\title{
Embedded Alamouti Space-Time Codes for High Rate and Low Decoding Complexity
}

\author{
Mohanned O. Sinnokrot, John R. Barry and Vijay K. Madisetti \\ Georgia Institute of Technology, Atlanta, GA 30332 USA, \{mohanned.sinnokrot@, barry@ece., vkm@ece.\}gatech.edu
}

\begin{abstract}
We introduce a new family of space-time codes called embedded Alamouti space-time (EAST) codes which offer high rate, a nonvanishing determinant and low decoding complexity. The family is parameterized by both the number of transmit antennas, which can range from two to eight, and by the rate, which can range from one to half the number of transmit antennas. The EAST codes combine a modified version of the perfect spacetime codes with an Alamouti embedding. For rates higher than one, the resulting space-time codes are simultaneously lower in decoding complexity and better performing than all known previous constructions in terms of the error probability achieved on a quasistatic Rayleigh fading channel with a given dimension.
\end{abstract}

Index Terms - transmit diversity, space-time coding.

\section{INTRODUCTION}

Recent work in space-time code design has shifted away from increasing the diversity order [1,2] or transmission rate [3] alone to increasing both simultaneously [4-9]. The continuous tradeoff between the achievable diversity and multiplexing gains as a function of the signal-to-noise ratio (SNR) was characterized in [10]. The threaded algebraic space-time (TAST) codes of [4] achieve the extremal points of the diversity-multiplexing tradeoff for any number of antennas and arbitrary rates. The perfect space-time codes of [7] achieve the entire frontier of the diversity-multiplexing tradeoff. Perfect space-time codes were originally proposed for only two, three, four and six antennas in [7] and later generalized for any number of antennas in [8].

One approach to constructing a high-rate code is to start with a maximal-rate TAST or perfect space-time code and then puncture one or more of its layers. For example, TAST and perfect space-time codes of rate $R \in\{1,2,3,4\}$ are easily obtained by puncturing $4-R$ layers of a rate- 4 code. Unfortunately, the resulting codes often have a high decoding complexity and worse BER performance than space-time codes that were designed for a particular rate and a particular number of antennas.

No previously reported space-time code achieve an arbitrary rate for arbitrary number of antennas while maintaining low decoding complexity. In this paper we partially fill this gap by proposing a framework for the construction and decoding of high rate space-time block codes. In particular, we make three contributions. First, we show that the complex rotation matrices of the perfect space-

This research was supported in part by a grant from Texas Instruments. time code of [7] can be replaced by equivalent real-valued matrices without affecting the diversity or coding gain; the real matrices lead to lower-complexity decoding. Second, we use the equivalent real generator matrices to construct the embedded Alamouti space-time (EAST) codes, which is a family of codes for any number of antennas up to eight, and for any rate up to half the number of antennas. When compared to previously reported codes with the same number of antennas and the same rate larger than one, the EAST codes are simultaneously lower in complexity and lower in error probability. Lastly, we show that the proposed code subsumes the rate-one semi-orthogonal algebraic spacetime (SAST) codes of [11] as a special case.

The remainder of the paper is organized as follows. In Section II, we present the system model, review the design criteria of space-time codes, and review the construction of the perfect, TAST and SAST codes. In Section III, we present the equivalent real generator matrix of the perfect space-time code. In Section IV, we present the proposed family of embedded Alamouti space-time codes, and we describe their decoder. In Section V, we present numerical results. In Section VI, we conclude the paper.

\section{System Model AND BACKGROUND}

\section{A. System Model and Code Design Criteria}

We consider a space-time code with $M$ transmit antennas transmitting $P$ complex information symbols over $T \in\{M$, $M+1\}$ symbol periods, so that the rate of the space-time code is $R=P / T$ symbols per channel use. The transmitted codeword can be expressed as a $T \times M$ matrix:

$$
\mathbf{C}=\left[\begin{array}{cccc}
c_{1}[1] & c_{2}[1] & \cdots & c_{M}[1] \\
c_{1}[2] & c_{2}[2] & & c_{M}[2] \\
\vdots & \vdots & & \vdots \\
c_{1}[T] & c_{2}[T] & \cdots & c_{M}[T]
\end{array}\right]
$$

where $c_{m}[t]$ denotes the symbol transmitted from antenna $m \in\{1, \ldots M\}$ at time $t \in\{1, \ldots T\}$. The received signal $y_{n}[t]$ at receive antenna $n \in\{1, \ldots N\}$ at time $t$ is:

$$
y_{n}[t]=\sum_{m=1}^{M} h_{m, n} c_{m}[t]+w_{n}[t],
$$

where $w_{n}[t]$ is the complex AWGN at receive antenna $n$ at time $t$, and $h_{m, n}$ is the channel coefficient between the $m$-th transmit antenna and $n$-th receive antenna. We assume quasistatic fading, so that $h_{m, n}$ is independent of time.

The maximum spatial diversity order for the $M$-input $N$ output channel is the product $M N$. The following two design 
criteria for space-time codes over quasistatic fading channels were derived in [2]:

- Rank Criterion: To achieve a full diversity of $M N$, the difference matrix $\Delta \mathbf{C}=\mathbf{C}-\tilde{\mathbf{C}}$ must have full rank for any pair of distinct codewords $\mathbf{C}$ and $\tilde{\mathbf{C}}$.

- Determinant Criterion: To further optimize performance, a code with full diversity should be chosen to maximize the asymptotic coding gain:

$$
\Gamma=\min _{\mathbf{C} \neq \tilde{\mathbf{C}}} \operatorname{det}\left((\mathbf{C}-\tilde{\mathbf{C}})^{*}(\mathbf{C}-\tilde{\mathbf{C}})\right)^{1 / M} .
$$

\section{B. Review of the Perfect, TAST and SAST Codes}

We begin with a review of the perfect [7], TAST [4], and SAST [11] codes. The perfect codes were originally designed as full-rate codes for square channels, but with puncturing, they can achieve any rate in the range $R \in\{1, \ldots, M\}$. The rate of the TAST codes is limited to the same range and the rate of the SAST codes is always $R=1$.

Let $\mathbf{a}_{\ell}=\left[a_{\ell, 1}, a_{\ell, 2}, \ldots, a_{\ell, M}\right]^{T}$ denote the vector of $M$ information symbols for the $\ell$-th thread, $\ell \in\{1, \ldots, R\}$, where $a_{\ell, m}$ is a complex symbol drawn from a $q$-ary QAM or HEX alphabet. A QAM alphabet is a subset of $\mathbb{Z}[i] \triangleq$ $\{u+v i, u, v \in \mathbb{Z}\}$, where $i=\sqrt{-1}$, while a HEX alphabet is a subset of $\mathbb{Z}[j] \triangleq\{u+v j, u, v \in \mathbb{Z}\}$, where $j=e^{i 2 \pi / 3}$.

Let $\mathbf{G}_{M}$ denote an $M \times M$ real or complex unitary rotation or generator matrix, and let $\mathbf{e}_{m}$ denote the $m$-th column of the $M \times M$ identity matrix. Finally, let $\mathbf{D}_{\ell}=\operatorname{diag}\left(\mathbf{G}_{M} \mathbf{a}_{\ell}\right)$ be the diagonal matrix with diagonal elements consisting of a rotated version of the $\ell$-th symbol vector.

In terms of these definitions, the perfect space-time code [7], TAST [4] and SAST [11] codes are defined as follows.

- The rate- $R$ perfect space-time code for an $M$-input $N$ output MIMO system is [7]:

$$
\mathbf{C}_{\text {PERFECT }}=\sum_{\ell=1}^{R} \mathbf{D}_{\ell} \mathbf{J}^{\ell-1},
$$

where $\mathbf{J}=\left[\gamma \mathbf{e}_{M}, \mathbf{e}_{1}, \ldots, \mathbf{e}_{M-2}, \mathbf{e}_{M-1}\right]$ and $\gamma$ is a unitmagnitude complex number that ensures full diversity. When $M \in\{2,4\}$, then $\gamma=i$ and the alphabet is QAM; when $M=3$ or $M=6$, then $\gamma=j$ or $\gamma=-j$, respectively, and the alphabet is HEX.

- The rate- $R$ TAST code for an $M$-input $N$-output MIMO system is [4]:

$$
\mathbf{C}_{\mathrm{TAST}}=\sum_{\ell=1}^{R} \mathbf{D}_{\ell}(\gamma \mathbf{U})^{\ell-1},
$$

where $\mathbf{U}=\left[\mathbf{e}_{M}, \mathbf{e}_{1}, \ldots, \mathbf{e}_{M-2}, \mathbf{e}_{M-1}\right]$, and $\gamma$ is a unitmagnitude complex number that ensures a full-diversity TAST code. The value $\gamma$ depends on both the modulation alphabet size and number of transmit antennas $M$.

- An equivalent form of the rate-one SAST code for $M$ transmit antennas is [11]:

$$
\mathbf{C}_{\mathrm{SAST}}=\left[\begin{array}{rr}
\mathbf{D}_{1} & \mathbf{D}_{2} \\
-\mathbf{D}_{2}^{*} & \mathbf{D}_{1}^{*}
\end{array}\right],
$$

where $\mathbf{D}_{\ell}=\operatorname{diag}\left(\mathbf{G}_{M / 2} \mathbf{a}_{\ell}\right)$ is a diagonal algebraic space-time (DAST) codeword for $M / 2$ antennas and $M / 2$ information symbols $\mathbf{a}_{\ell}$. This version of SAST differs from the original in [11] only by the fact that the submatrices $\mathbf{D}_{\ell}$ are diagonal instead of circulant. The circulant matrix reduces the peak-to-average-power ratio (PAPR) but has no impact on the diversity, coding gain, and decoding complexity. We simply use a diagonal matrix in (6) to simplify the presentation, and to better align with our newly proposed codes.

\section{Perfect Codes with Real Generators}

We now show that we can replace the complex generator matrices of the perfect codes of [7] for two, three and four antennas with a real generator matrix without affecting the diversity or coding gain.

We next examine separately the structure of the generator matrix for the perfect code of [7] for the special cases of $M=1, M=2, M=3$, and $M=4$ antennas, respectively.

- When $M=1$, the perfect code is equivalent to no code at all, with the scalar identity generator:

$$
\mathbf{G}_{1}=1 \text {. }
$$

- When $M=2$, the perfect code reduces to the golden code [5], whose generator is equivalent to the following real generator matrix (obtained independently in [6]):

$$
\mathbf{G}_{2}=\left[\begin{array}{rr}
\alpha_{1} & \alpha_{2} \\
-\alpha_{2} & \alpha_{1}
\end{array}\right]
$$

where $\alpha_{1}=\cos (\theta), \alpha_{2}=\sin (\theta)$ and $\theta=\frac{1}{2} \tan ^{-1}(2)$.

- When $M=3$, the perfect-code generator matrix can be written as $\mathbf{G}_{3}^{c}=\mathbf{G}_{3} \mathbf{X}$, where

$$
\mathbf{G}_{3}=\frac{1}{\sqrt{7}}\left[\begin{array}{rrr}
\left|\alpha_{1}\right| & \left|\alpha_{2}\right| & \left|\alpha_{3}\right| \\
-\left|\alpha_{2}\right| & -\left|\alpha_{3}\right| & \left|\alpha_{1}\right| \\
\left|\alpha_{3}\right| & -\left|\alpha_{1}\right| & -\left|\alpha_{2}\right|
\end{array}\right],
$$

and $\mathbf{X}=\operatorname{diag}\left(\left[e^{i \measuredangle \alpha_{1}}, e^{i \measuredangle \alpha_{2}}, e^{i \measuredangle \alpha_{3}}\right]\right), \alpha_{1}=1+j+\tau$, $\alpha_{2}=-1+j+\tau^{2}, \alpha_{3}=-1 / \tau+j$, and $\tau=2 \cos (2 \pi / 7)$.

- When $M=4$, the perfect-code generator matrix can be written as $\mathbf{G}_{4}^{c}=\mathbf{Y} \mathbf{G}_{4}$, where

$$
\begin{aligned}
& \mathbf{G}_{4}=\frac{1}{\sqrt{15}}\left[\begin{array}{rrrr}
\left|\alpha_{1}\right| & \beta\left|\alpha_{2}\right| & -\beta\left|\alpha_{1}\right| & -\left|\alpha_{2}\right| \\
\beta\left|\alpha_{4}\right| & \left|\alpha_{3}\right| & \left|\alpha_{4}\right| & \beta\left|\alpha_{3}\right| \\
\left|\alpha_{2}\right| & -\beta\left|\alpha_{1}\right| & -\beta\left|\alpha_{2}\right| & \left|\alpha_{1}\right| \\
\beta\left|\alpha_{3}\right| & -\left|\alpha_{4}\right| & \left|\alpha_{3}\right| & -\beta\left|\alpha_{4}\right|
\end{array}\right], \\
& \text { and } \mathbf{Y}=\operatorname{diag}\left(\left[e^{i \measuredangle \alpha_{1}}, e^{i \measuredangle \alpha_{3}}, e^{i \measuredangle \alpha_{1}}, e^{i\left(\measuredangle \alpha_{3}-\pi / 2\right)}\right]\right) \text {, } \\
& \alpha_{1}=1-4 \tau i+i \tau^{3}, \alpha_{2}=1+i(\tau-1), \alpha_{3}=(1-3 i) \tau
\end{aligned}
$$


$+i \tau^{3}, \alpha_{4}=-i+(-3+4 i) \tau+(1-i) \tau^{3}, \beta=(1+$ $\sqrt{5}) / 2$, and $\tau=2 \cos (2 \pi / 15)$.

Theorem 1. If the entries of $\mathbf{v}$ are drawn from $\mathbb{Z}[j]$, then $\operatorname{diag}\left(\mathbf{G}_{3}^{c} \mathbf{v}\right)$ and $\operatorname{diag}\left(\mathbf{G}_{3} \mathbf{v}\right)$ have the same coding gain and diversity.

Theorem 2. If the entries of $\mathbf{v}$ are drawn from $\mathbb{Z}[i]$, then $\operatorname{diag}\left(\mathbf{G}_{4}^{c} \mathbf{v}\right)$ and $\operatorname{diag}\left(\mathbf{G}_{4} \mathbf{v}\right)$ have the same coding gain and diversity.

We omit the proofs for brevity.

\section{The Proposed Embedded Alamouti Codes}

\section{A. Encoding}

We will assume that the number of transmit antennas is even, falling in the range $M \in\{2,4,6,8\}$; the space-time code for an odd number of antennas is obtained by deleting the last column of a space-time code for $M+1$ antennas.

The rate- $R$ EAST code is constructed by combining a pair of rate- $R$ perfect space-time codes with real generators in a modified ABBA format.

Specifically, let $\quad \mathbf{P}(\mathbf{a})=\sum_{\ell=1}^{R} \operatorname{diag}\left(\mathbf{G}_{M / 2} \mathbf{a}_{\ell}\right) \mathbf{J}^{\ell-1}$ denote a rate- $R$ perfect space-time encoder for $M / 2$ antennas and $R M / 2$ information symbols $\mathbf{a}=\left[\begin{array}{lll}\mathbf{a}_{1}^{T}, \ldots & \mathbf{a}_{R}^{T}\end{array}\right]^{T}$, with real generator specified by either (7), (8), (9) or (10), where $\mathbf{J}=\left[\gamma \mathbf{e}_{M / 2}, \mathbf{e}_{1}, \ldots, \mathbf{e}_{M / 2-1}\right]$. The constant $\gamma$ and the modulation alphabet depends on $M$ : when $M=6$, the constant is $\gamma=j$ and the alphabet is HEX. Otherwise, the constant is $\gamma=i$ and the alphabet is QAM.

Similarly, let $\mathbf{P}(\mathbf{b})$ be a second such codematrix for an independent set of $R M / 2$ information symbols $\mathbf{b}$. The proposed rate- $R$ EAST code is then:

$$
\mathbf{C}_{\mathrm{EAST}}=\left[\begin{array}{ll}
\mathbf{P}(\mathbf{a}) & \mathbf{P}(\mathbf{b}) \\
\mathbf{P}\left(-\mathbf{b}^{*}\right) & \mathbf{P}\left(\mathbf{a}^{*}\right)
\end{array}\right]
$$

In theory, the maximum rate $R$ for the proposed EAST codes is $M / 2$. In practice, a rate of $R \leq \min (N, M / 2)$ will be needed to ensure reliable decoding at the receiver.

The modified ABBA construction of (11) is referred to as an Alamouti embedding, because it can be expressed as a linear dispersion code with the Alamouti dispersion matrices. The alternative form of the code is omitted for brevity but the Alamouti structure is readily apparent in (11); the information symbols a appear along with their conjugate and the information symbols $\mathbf{b}$ appear along with their negative conjugate. Furthermore, when the number of transmit antennas is $M=2$, the EAST code reduces to the original Alamouti code [1].

Proposition 1. Let the coding gain (3) of the perfect space-time code [7] for $M / 2$ transmit antennas with rate
$R \leq \min (N, M / 2)$ be $\Gamma=\lambda \cdot d_{\min }^{2}$, where $0<\lambda<1$, and where $d_{\min }$ is the minimum Euclidean distance of the $q$-QAM or $q$-HEX constellation. Then the coding gain of the proposed EAST code with the same rate but for $M$ transmit antennas is also given by $\lambda \cdot d_{\mathrm{min}}^{2}$.

We make several important remarks regarding the construction of the proposed space-time code.

- When the rate of the code is $R=1$, the EAST code simplifies to the equivalent form of the SAST code given in (6). The proposed code thus has the same diversity, coding gain, and decoding complexity as the SAST code when $R=1$, albeit with a larger PAPR.

- The EAST code has twice has many layers as the perfect and TAST codes. Specifically, the EAST code has $2 R$ layers, each layer encoding $M / 2$ symbols, for a total of $M R$ symbols. In contrast, the perfect and TAST codes encode $R$ layers, with $M$ symbols in each layer.

- Besides the number of layers, the EAST code also differs from the perfect and TAST codes because it encodes the symbols and their conjugate in each layer.

- The proposed EAST code differs from other ABBA codes in [11] and [12] in that the symbols are conjugated before encoding. In fact, if the code matrices $\mathbf{P}\left(\mathbf{a}^{*}\right)$ and $\mathbf{P}\left(-\mathbf{b}^{*}\right)$ are replaced with either the conjugate matrices $(\mathbf{P}(\mathbf{a}))^{*}$ and $(\mathbf{P}(-\mathbf{b}))^{*}$ respectively, as done in [12], or with the conjugate transpose matrices $(\mathbf{P}(\mathbf{a}))^{\mathcal{H}}$ and $(\mathbf{P}(-\mathbf{b}))^{\mathcal{H}}$, respectively, as done in [11], then the codematrix in (11) is no longer full rank.

- The Alamouti embedding of (11) offers two advantages. First, when compared to the perfect space-time codes or TAST codes, the proposed codes have a few number of zeros in the space-time code matrix, and hence, a lower PAPR. Second, Alamouti embedding reduces the decoding complexity because it allows the layers to be grouped in pairs, so that the two layers in a pair are separately decodable. This feature reduces both the worst-case and average decoding complexity.

\section{B. Decoding}

In order to ensure reliable detection at the receiver, we will assume that $N \geq R$. After conjugating the last $M / 2$ received samples, the received vector at the $n$-th receive antenna during time slots $t \in\{1, \ldots, M\}$ can be written as:

$$
\left[\begin{array}{c}
y_{n}[1] \\
\vdots \\
y_{n}[M / 2] \\
y_{n}^{*}[M / 2+1] \\
\vdots \\
y_{n}^{*}[M]
\end{array}\right]=\tilde{\mathbf{H}}_{n} \Omega\left[\begin{array}{c}
\mathbf{a}_{1} \\
\mathbf{b}_{1} \\
\vdots \\
\mathbf{a}_{R} \\
\mathbf{b}_{R}
\end{array}\right]+\left[\begin{array}{c}
w_{n}[1] \\
\vdots \\
w_{n}[M / 2] \\
w_{n}^{*}[M / 2+1] \\
\vdots \\
w_{n}^{*}[M]
\end{array}\right]
$$

where $\Omega=\mathbf{I}_{2 R} \otimes \mathbf{G}_{M / 2}, \otimes$ is the Kronecker product and 


$$
\tilde{\mathbf{H}}_{n}=\left[\begin{array}{ccccc}
\mathbf{H}_{1, n}(0) & \mathbf{H}_{1, n}(1) & \ldots & \mathbf{H}_{R, n}(0) & \mathbf{H}_{R, n}(1) \\
\mathbf{H}_{1, n}^{*}(1) & -\mathbf{H}_{1, n}^{*}(0) & \ldots & \mathbf{H}_{R, n}^{*}(1) & -\mathbf{H}_{R, n}^{*}(0)
\end{array}\right]
$$

where $\quad \mathbf{H}_{\ell, n}(k)=\operatorname{diag}\left(\mathbf{J}^{\ell-1} \mathbf{h}_{n}(k)\right)$

and $\mathbf{h}_{n}(k)=\left[h_{k M / 2+1, n}, h_{k M / 2+2, n}, \ldots, h_{k M / 2+M / 2, n}\right]^{T}$.

In matrix form, (12) can be expressed as follows:

$$
\mathbf{Y}_{n}=\tilde{\mathbf{H}}_{n} \Omega \mathbf{S}+\mathbf{W}_{n} .
$$

Stacking the received vectors from the $N$ antennas yields:

$$
\overline{\mathbf{Y}}=\left[\begin{array}{c}
\mathbf{Y}_{1} \\
\vdots \\
\mathbf{Y}_{N}
\end{array}\right]=\left[\begin{array}{c}
\tilde{\mathbf{H}}_{1} \\
\vdots \\
\tilde{\mathbf{H}}_{N}
\end{array}\right] \Omega \mathbf{S}+\left[\begin{array}{c}
\mathbf{W}_{1} \\
\vdots \\
\mathbf{W}_{N}
\end{array}\right]=\overline{\mathbf{H}} \mathbf{S}+\overline{\mathbf{W}} .
$$

The maximum-likelihood receiver decides on the transmitted symbol vector $\mathbf{S}$ that minimizes $\|\overline{\mathbf{Y}}-\overline{\mathbf{H}} \mathbf{S}\|^{2}$. Let $\overline{\mathbf{H}}=\mathbf{Q R}$ be a Q-R decomposition, where $r_{l, p}=\mathbf{q}_{l}{ }^{*} \mathbf{h}_{p}$ is the inner product between the $l$-th column of $\mathbf{Q}$ and the $p$-th column of $\overline{\mathbf{H}}$. The ML decoding of (15) is equivalent to minimizing $\| \mathbf{V}$ $-\mathbf{R S} \|^{2}$, where $\mathbf{V}=\mathbf{Q}^{*} \overline{\mathbf{Y}}$. We next discuss properties of the $\mathbf{R}$ matrix that allows for reduced complexity decoding.

The following properties of the $\mathbf{R}$ matrix in the Q-R decomposition of the effective channel matrix $\overline{\mathbf{H}}$ can be verified by direct computation, where $L=M / 2$ :

(a) $r_{l, p}=0, l \in\{1, \ldots L\}, p \in\{L+1, \ldots 2 L\}$ and $R \in\{1, \ldots 4\}$

(b) $r_{l, p}=0, l \in\{2 L+1, \ldots 3 L\}, p \in\{3 L+1, \ldots 4 L\}$ and $R \in\{2, \ldots 4\}$

(c) $r_{l, p}=0, l \in\{4 L+1, \ldots 5 L\}, p \in\{5 L+1, \ldots 6 L\}$ for $R \in\{3,4\}$

(d) $r_{l, p}=0, l \in\{6 L+1, \ldots 7 L\}, p \in\{7 L+1, \ldots 8 L\}$ for $R=4$

(e) $r_{l, p} \in \mathbb{R}, \quad l, p \in\{1, \ldots, L\}, \quad$ and $l, p \in\{L+1, \ldots 2 L\}$, for $R \in\{1, \ldots 4\}$

(f) $r_{l, p} \in \mathbb{R}, \quad l, p \in\{2 L+1, \ldots 3 L\}$ $l, p \in\{3 L+1, \ldots 4 L\}$ for $R \in\{2, \ldots 4\}$

(g) $r_{l, p} \in \mathbb{R}, \quad l, p \in\{4 L+1, \ldots 5 L\}$ $l, p \in\{5 L+1, \ldots 6 L\}$ for $R \in\{3,4\}$

(h) $r_{l, p} \in \mathbb{R}, \quad l, p \in\{6 L+1, \ldots 7 L\}$ $l, p \in\{7 L+1, \ldots 8 L\}$ for $R=4$.

The first four properties (a) - (d) follow directly from the orthogonality of the corresponding columns in $\overline{\mathbf{H}}$. The last four properties (e) - (h) follow from using a real rotation matrix to encode each layer.

In determining the worst-case decoding complexity, it is only necessary to consider properties (a) and (e). This is because the proposed EAST code is not separable and the reduction in the worst-case complexity is determined by the reduction in decoding complexity of the last decoded layer. and
TABLE I. WORST-CASE DECODING COMPLEXITY FOR SEVERAL CODES.

\begin{tabular}{|c|c||c|c|c|c|c|}
\hline \#ants. & Rate & $\begin{array}{c}\text { Perfect } \\
{[7]}\end{array}$ & TAST [4] & $\begin{array}{c}\text { Q-Orth. } \\
{[12]}\end{array}$ & $\begin{array}{c}\text { SAST } \\
{[11]}\end{array}$ & Proposed \\
\hline \hline$M=2$ & $R=1$ & $2 q$ & $2 q$ & - & $4 \sqrt{q}$ & $4 \sqrt{q}$ \\
\hline \multirow{3}{*}{$M=4$} & $R=1$ & $2 q^{2}$ & $2 q^{2}$ & $2 q^{2}$ & $4 q$ & $4 q$ \\
\cline { 2 - 7 } & $R=2$ & $2 q^{6}$ & $2 q^{6}$ & - & - & $4 q^{5}$ \\
\hline \multirow{4}{*}{$M=6$} & $R=1$ & $q^{6}$ & $2 q^{3}$ & $2 q^{3}$ & $4 q^{1.5}$ & $4 q^{1.5}$ \\
\cline { 2 - 7 } & $R=2$ & $q^{12}$ & $2 q^{9}$ & - & - & $4 q^{8}$ \\
\cline { 2 - 7 } & $R=3$ & $q^{18}$ & $2 q^{15}$ & - & - & $4 q^{14}$ \\
\hline \multirow{4}{*}{$M=8$} & $R=1$ & $2 q^{4}$ & $2 q^{4}$ & $2 q^{4}$ & $4 q^{2}$ & $4 q^{2}$ \\
\cline { 2 - 7 } & $R=2$ & $2 q^{12}$ & $2 q^{12}$ & - & - & $4 q^{10}$ \\
\cline { 2 - 7 } & $R=3$ & $2 q^{20}$ & $2 q^{20}$ & - & - & $4 q^{18}$ \\
\cline { 2 - 7 } & $R=4$ & $2 q^{28}$ & $2 q^{28}$ & - & - & $4 q^{26}$ \\
\hline
\end{tabular}

Due to property (a), the symbols $\mathbf{a}_{1}$ can be decoded separately from the symbols $\mathbf{b}_{1}$. Due to property (e), the real components of the vector $\mathbf{a}_{1}$ and $\mathbf{b}_{1}$ are separately decodable from the imaginary components. As a result, the worst-case decoding complexity is $4 q^{M / 4}$ for any layer in the proposed space-time code for $q$-QAM alphabet. For $q$-HEX alphabet ( $M=6, R \geq 2)$, coordinate interleaving prior to encoding can be used to reduce the worst-case complexity. The worstcase complexity of the perfect, TAST, quasiorthogonal, SAST, and proposed EAST code is summarized in Table I.

We note that when the number of transmit antennas $M$ is odd, the decoding steps are identical to the case of $M+1$ antennas, with the exception that the channel coefficients for the $(M+1)$-th antenna are simply set to zero in (15).

\section{Numerical Results}

In this section we compare the BER performance and complexity of the proposed space-time codes with the best performing space-time codes of [4, 7-12] over quasistatic Rayleigh-fading channel with additive Gaussian noise. As a representative example, we compare BER and complexity for four transmit antennas and two receive antennas for $R=$ 2. In Fig. 1, we show BER as a function of SNR per bit for the proposed space-time code, TAST [4] code and the fastdecodable code (FAST) of [9]. At BER $=10^{-4}$, the proposed space-time code outperforms the fast-decodable code and TAST code by $0.3 \mathrm{~dB}$ and $1 \mathrm{~dB}$, respectively.

In Fig. 2 we show a plot of average node count as a function of SNR per bit for proposed EAST code, TAST and fast-decodable code. In all cases, the ML decoder was implemented using the complex sphere decoder with sorting, sphere radius update and depth-first tree search. For the proposed EAST code and fast-decodable code, the sphere decoding algorithm was implemented as a 4-level sphere decoder to decode the first layer, followed by four 1-level sphere decoders to decode the second layer, which has a 
worst-case decoding complexity of $4 q$, resulting in overall worst-case decoding complexity of $4 q^{5}$. For the TAST code, the sphere decoding algorithm was implemented as a 4-level sphere decoder to decode the first layer, followed by two 2level sphere decoders to decode the second layer, which has a worst-case complexity of $2 q^{2}$, resulting in overall worst-case decoding complexity of $2 q^{6}$. As can be seen from Fig. 2 , the average complexity of the proposed space-time code is about $30 \%$ and $20 \%$ less complex than the TAST and FAST codes.

We omit simulation results for other configurations due to lack of space but note that the EAST codes space-time codes of [4,7-12] except for the $R=1$ configuration, where the quasiorthogonal codes perform slightly better.

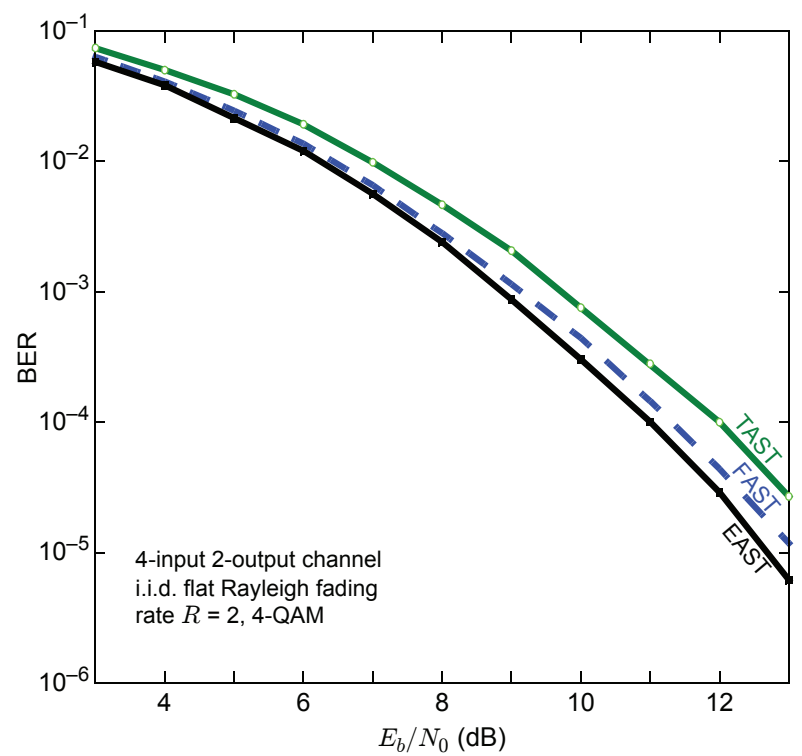

Fig. 1. Performance comparison.

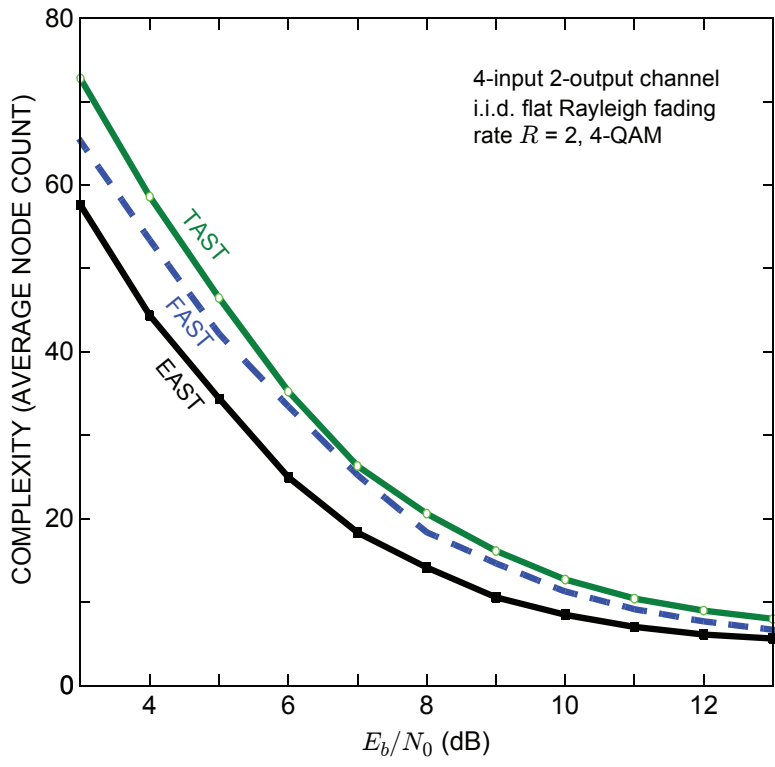

Fig. 2. Complexity comparison.

\section{CONCLUSIONS}

We have proposed a family of space-time codes for up to eight transmit antennas and rates up to half the number of transmit antennas. The proposed space-time codes were constructed using the real versions of the complex generator matrices of the perfect code; these equivalent generators offer the same diversity and coding gain but enable reducedcomplexity decoding. Alamouti embedding was used to not only obtain space-time codes with higher coding gain than the perfect codes, but also lower decoding complexity. The proposed construction is the first systematic construction of space-time codes with low-complexity decoding, not only for rate one, but for rates up to half the number of transmit antennas. Furthermore, the proposed space-time codes outperform all previous constructions with the same number antennas, diversity gain and rate for rates higher than one.

\section{REFERENCES}

[1] S. M. Alamouti, "A Simple Transmit Diversity Technique For Wireless Communications," IEEE J. Sel. Areas Commun., vol. 16, pp. 1451-1458, Oct. 1998.

[2] V. Tarokh, N. Seshadri, A. Calderbank, "Space-Time Codes For High Data Rate Wireless Communication: Performance Criterion And Code Construction," IEEE Trans. IT, 44, pp. 744-, Mar. 1998.

[3] P.W. Wolniansky, G.J. Foschini, G.D. Golden, R.A. Valenzuela, "VBLAST: An Architecture for Realizing Very High Data Rates Over The Rich Scattering Wireless Channel," ISSSE 98, p. 295-300, 1998.

[4] M. O. Damen, H. E. Gamal and N. C. Beaulieu, "Linear Threaded Algebraic Space-Time Constellations," IEEE Trans. Inf. Theory, vol. 49, pp. 2372 - 2388, Oct. 2003

[5] J.-C. Belfiore, G. Rekaya, and E. Viterbo, "The Golden Code: A $2 \times 2$ Full Rate Space-Time Code with Nonvanishing Determinants," IEEE Trans. on Inf. Theory, vol. 51, no 4, Apr. 2005.

[6] P. Dayal and M. K. Varanasi, "An Optimal Two Transmit Antenna Space-Time Code And Its Stacked Extensions," IEEE Trans. Inf. Theory, vol. 51, n. 12, pp. 4348-4355, Dec. 2005.

[7] F. Oggier, G. Rekaya, J.-C. Belfiore, and E. Viterbo, "Perfect SpaceTime Block Codes," IEEE Trans. IT, 52, n. 9, pp. 3885-, Sep. 2006.

[8] P. Elia, B. A. Sethuraman and P. Kumar, "Perfect Space-Time Codes with Minimum And Non-Minimum Delay For Any Number of Transmit Antennas", IEEE Trans. IT, 53, n. 11, pp. 3853-, Nov. 2007.

[9] E. Biglieri, Y. Hong, and E. Viterbo, "On Fast Decodable SpaceTime Block Codes," submitted Aug. 2007.

[10] L. Zheng and D. Tse, "Diversity and Multiplexing: A Fundamental Tradeoff in Multiple Antenna Channels," IEEE Trans. on Inf. Theory, vol. 49, no 4, pp. 1073-1096, May 2003.

[11] D. N. Dao, C. Yuen, C. Tellambura, Y. L. Guan and T. T. Tjhung, "Four-Group Decodable Space-Time Block Codes," IEEE Trans. Signal Processing, vol. 56, Jan. 2008, Pages: 424-430.

[12] N. Sharma and C. Papadias, "Full Rate Full Diversity Linear QuasiOrthogonal Space-Time Codes For Any Transmit Antennas," EURASIP J. Applied Sign.Processing, no. 9, pp. 1246-, Aug. 2004.

[13] J. Boutros and E. Viterbo, "Signal Space Diversity: A Power- And Bandwidth-Efficient Diversity Technique For The Rayleigh Fading Channel," IEEE Trans. on IT, 44, no. 4, pp. 1453-1467, Jul. 1998. 